Review

\title{
Image Guided Biodistribution and Pharmacokinetic Studies of Theranostics
}

\author{
Hong Ding ${ }^{1,2}{ }^{\bowtie}$, Fang $\mathrm{Wu}^{2}$ \\ 1. Department of Immunology, Herbert Wertheim College of Medicine, Florida International University, Miami, FL33199. \\ 2. Department of Pharmaceutical Sciences, University at Buffalo, The State University of New York, Buffalo, New York 14260.
}

\begin{abstract}
$\triangle$ Corresponding author: Hong Ding, Department of Immunology, Florida International University, Miami, FL33199. Tel: 305-348-1490, Fax: 305-348-1109. Email: hding@fiu.edu.

(c) Ivyspring International Publisher. This is an open-access article distributed under the terms of the Creative Commons License (http://creativecommons.org/ licenses/by-nc-nd/3.0/). Reproduction is permitted for personal, noncommercial use, provided that the article is in whole, unmodified, and properly cited.
\end{abstract}

Received: 2012.05.24; Accepted: 2012.06.17; Published: 2012.11.05

\begin{abstract}
Image guided technique is playing an increasingly important role in the investigation of the biodistribution and pharmacokinetics of drugs or drug delivery systems in various diseases, especially cancers. Besides anatomical imaging modalities such as computed tomography (CT), magnetic resonance imaging (MRI), molecular imaging strategy including optical imaging, positron emission tomography (PET) and single-photon emission computed tomography (SPECT) will facilitate the localization and quantization of radioisotope or optical probe labeled nanoparticle delivery systems in the category of theranostics. The quantitative measurement of the bio-distribution and pharmacokinetics of theranostics in the fields of new drug/probe development, diagnosis and treatment process monitoring as well as tracking the brain-blood-barrier (BBB) breaking through by high sensitive imaging method, and the applications of the representative imaging modalities are summarized in this review.
\end{abstract}

Key words: Image, biodistribution, pharmacokinetic, theranostics, nanoparticles, antibody.

\section{Introduction}

The properties of biodistribution and pharmacokinetics play a major role in influencing and determining the efficacy and safety for the treatment with a medicine. Currently, several image guided modalities have been applied in biomedicine and even in clinic, including magnetic resonance imaging (MRI), X-ray computed tomography (CT), positron emission tomography (PET), single-photon emission computed tomography (SPECT), electron microscopy, autoradiography, optical imaging and ultrasound (US), etc. They are all noninvasive imaging modalities and proven clinical applications, and some of them are only extended to in-vivo research specimens as small as mice [1, 2]. Among them, PET and optical imaging are regarded as quantitative or semi-quantitative imaging modalities that employ radiotracers or optical tracers to image biodistribution of the labeled drugs or probe loaded delivery systems in the body, meanwhile CT and MRI are normally used for anatomical imaging purposes $[3,4]$. In these imaging modalities, non-invasive technique attracted more attention because of its properties of no breaking in the skin and no contact with the mucosa, internal body cavity beyond a natural or artificial body orifice. Non-invasive in vivo molecular imaging can be obtained from PET, MR, CT and visible infrared in vivo optical imaging systems. The discovery of the first modern non-invasive techniques was setup at the end of $19^{\text {th }}$ century based on physical methods: electrocardiography and X-rays. From that time, non-invasive with advantage of penetrating the body rather than a scalpel have continuously enlarged the 
scope of medical technology. Non-invasive techniques commonly can be used for the purpose of theranostics by diagnostic imaging and therapy on diseases [5-7].

The ability to quantitatively image the biodistribution of therapeutics or drug delivery systems in a noninvasive manner can aid in the development of new theranostic, dose optimization and treatment monitoring. Normally, the information of biodistribution can often be obtained by dissecting the animal, collecting plasma or tissues and being analyzed by high-performance liquid chromatography (HPLC), Enzyme-linked immunosorbent assay (ELISA) etc [8-10], the key advantage of non-invasive imaging is less time consuming and more cost effective of animals and analysis reagents [11]. There is a critical need to establish an effective non-invasive tool to clearly diagnose diseases along with the treatment. If a high effective non-invasive method exists, a cancer patient could be more potentially received effective treatment at an earlier stage with concise targeted drug delivery [12].

Therefore, discussions of perspectives of various imaging guided modalities are focusing on the re- search applications in biodistribution and pharmacokinetics of delivery systems such as theranostics. The concept of the revolutionary "Theranostic" was originated by Funkhouser in 2002 from one of his reviews. Theranotics is defined as a material that combines the modalities of therapy and diagnostic imaging at the same time within the same dose [13]. It integrates the two features into one "package" to overcome undesirable difference between biodistribution and therapeutic agents. The final goal of the theranostic is to donate materials with the capacity of monitoring the treated tissue, PK and efficacy in the long-term period. This approach will benefit nanomedicine with an effectively style of personalized treatment. In actual applciations, the combination of MRI, CT, PET, or optical imaging and their combination usage may add further impetus to the rapid and high throughput screening of nanoparticle delivery systems based diagnosis and therapeutics (Figure 1). Some representative applications of imaging modalities in the area of biodistribution and pharmacokinetics of nanoparticles for the aim of treatment and diagnosis are also listed in this review.

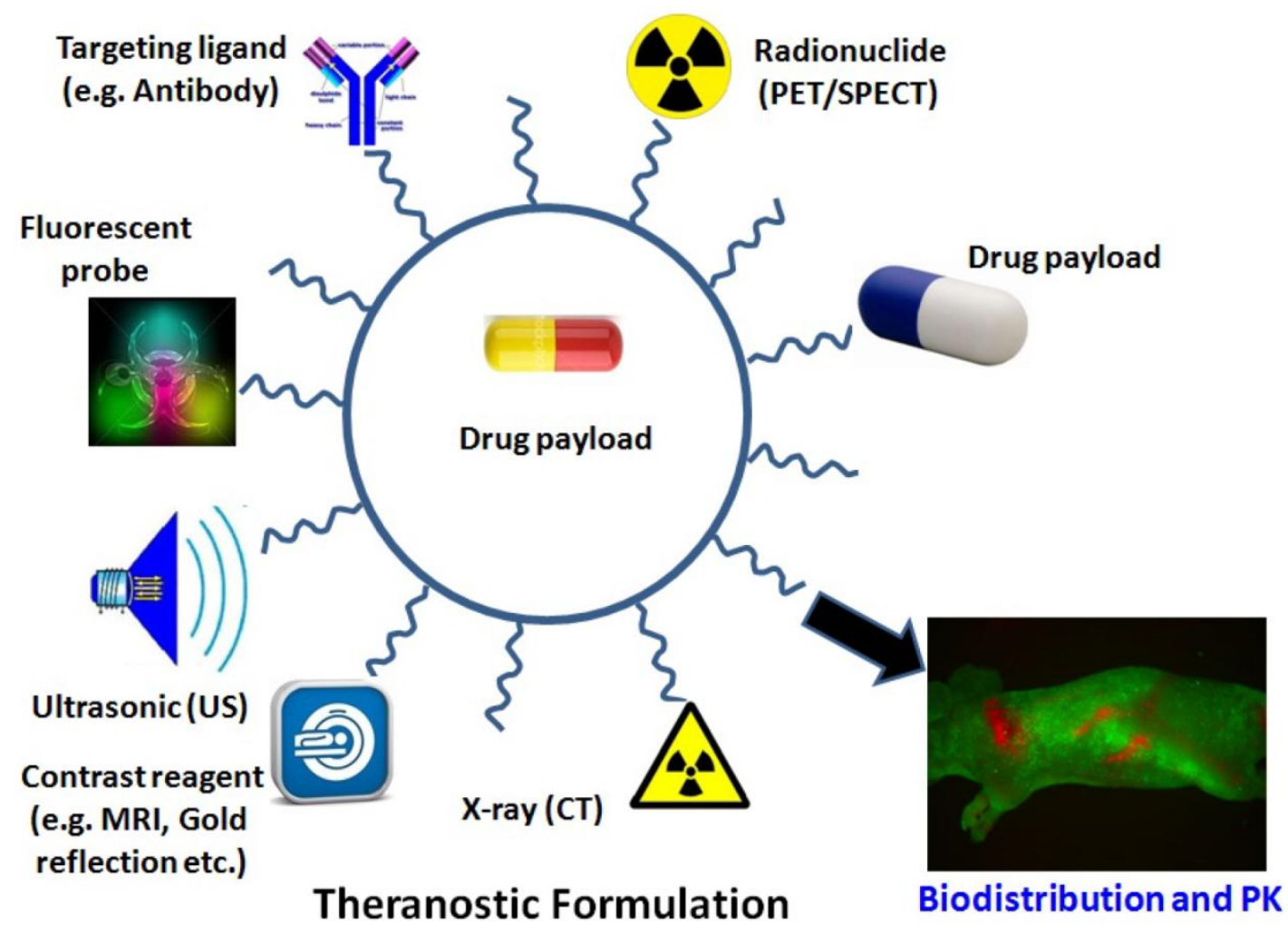

Figure I. Illustration of multi-functionalized nanoparticles. Examples of multifunctional nanoparticles for (i) molecular imaging for MRI or PET/SPECT imaging; (ii) drug delivery, (iii) fluorescence detection with a fluorescent probe; (iv) X-ray images such as CT; (v) ultrasonic assistance; (vi) specific targeting, functionalized with specific ligand molecules (e.g. antibody) and (vii) drug payload. 


\section{Magnetic resonance imaging (MRI) and computed tomography (CT) guided bio- distribution and pharmacokinetics study}

Magnetic resonance imaging (MRI) is a non-invasive medical imaging to visualize detailed internal structures. For MR imaging, atoms in the body are aligned under a powerful magnetic field. Radio frequency fields change the alignment of the magnetization of atoms. This causes the nuclei to produce a rotating magnetic field detectable by the scanner, which can be recorded to construct an image of the body. Strong magnetic field gradients cause nuclei at different locations to rotate at different speeds, thus providing 3D spatial information. Comparing with other medical imaging techniques such as computed tomography (CT), MRI provides good contrast between the different soft tissues of the body, which make it especially useful in imaging brain, muscles, the heart, and cancers [14, 15]. Another advantage of MR imaging is that it does not need to use ionizing radiation which has damage to cell structure once be exposed.

X-Rays computed tomography, also called computed tomography (CT scan) or computed axial tomography (CAT scan), can make use of radiation to get an internal view of the body for medical imaging employing tomography created by computer processing. Although CT technique is regarded as birth defects or DNA altering because of powerful X-ray radiation exposure, it is still largely employed in hospitals, especially used for broken bones and skulls. Not like MRI imaging which can provide subtle differences between the different kinds of soft tissues, CT can demonstrate the difference between bone density and soft tissue. Although MRI and X-ray are both imaging techniques for organs of the body, the difference in application is that MRI images provide a 3D representation of organs, which X-Rays usually cannot.

Dr. Sgouros and colleagues applied MRI based preclinical biodistribution and localization data and the therapeutic potential of trastuzumab monoclonal antibody (mAb) (anti-HER2/neu) against ovarian carcinoma. By MRI images, they found out the rapid blood-pool uptake (5-9 hours) of mAb after intraperitoneal injection followed by tumor localization (26-32 hours). And micro-PET finding was consistent with the data from high resolution MRI images slices for $\mathrm{mAb}$ bio-distribution in main organs of tumor-beard mice [16]. Dr. Teng et al conjugated anti-mouse OxLDL polyclonal antibody to polyethylene glycol-coated ultrasmall superparamagnetic iron oxide (USPIO) nanoparticles, and imaged at 7.0 Tesla MRI on a carotid perivascular collar model in atherosclerotic disease mice. The results showed MRI signal loss in the carotid atherosclerotic lesions after administration of targeted anti-OxLDL-USPIO at 8th hour and 24th hour, which means that anti-mouse OxLDL antibody conjugated magnetic nanoparticle can be used as a strategy for the therapeutic evaluation of atherosclerotic plaques in vivo by MRI imaging [17].

CT became an important tool in medical imaging to supplement $\mathrm{X}$-rays and medical ultrasonography since 1970s. It has more recently been used for preventive medicine or screening for disease. CT is regarded as a moderate- to high-radiation diagnostic technique. The radiation dose for a particular study depends on multiple factors: volume scanned, patient build, number and type of scan sequences, and desired resolution and image quality. Normally, the high resolution and image quality means the high exposure of radiation to patients. The high ionizing radiation in CT application can damage the DNA double strand structure in human body. In one study, researchers found out at a typical CT scanning, $40 \%-100 \%$ of the irradiated cells is damaged by one or more double strand breaks in DNA molecule. Although there is repair process in cells for damaged and broken DNA, the results are not satisfied [18]. That's why by now, CT technique is designed to combine with other image techniques for better application in patients.

\section{PET and SPECT image guided biodis- tribution and pharmacokinetics study of theranostics}

\section{I Radiolabeling of antibody therapeutics and principle of PET imaging}

PET imaging is another kind of non-invasive option for diagnosis. Since biodistribution of antibody-based therapeutics is a key consideration that can be modulated to impact the ensuing in vivo pharmacological effect. After being labeled with radionuclide of sufficient long half-life, antibody therapeutics can be monitored using PET (positron emission tomography) imaging with respect to pharmacokinetics and its biodistribution. Normally, it is assumed that the labeling method does not perturb the property of the molecules with respect to metabolism, specific binding or non-specific interactions. The labeling isotopes include positron emitters such as ${ }^{11} \mathrm{C}$, ${ }^{15} \mathrm{O},{ }^{18} \mathrm{~F},{ }^{68} \mathrm{Ga},{ }^{64} \mathrm{Cu}$, or ${ }^{76} \mathrm{Br}$ with half-lives of 20 minutes, 2 minutes, 2 hours, 68 minutes, 13 hours, and 16 hours respectively. Long-lived positron emitters such as $89 \mathrm{Zr}$ and $124 \mathrm{I}$ with half-lives of several days, 3.2 days for ${ }^{89} \mathrm{Zr}$ and 4.2 days for ${ }^{124} \mathrm{I}$, are also available 
[19]. Among them, Copper-64 $\left({ }^{64} \mathrm{Cu}\right)$ is a promising PET radionuclide, particularly for antibody-targeted imaging [20]. The reported methods for modification include addition of labeled groups or radiolabel chelating groups. Compared with radio-halogenation, radiometal ion complexation reactions have simpler chemistry and allow tracer production kits. For example, chelator 1, 4, 7, 10-tetraazacyclododecane-1, 4, 7,10 -tetraacetic acid (DOTA) is commonly used in radiolabeling copper radioisotope with antibody [21].

'MicroPET' is a small-animal positron emission tomography which uses pharmacologically or biochemically active compounds labeled with short-lived or long-lived positron-emitting radionuclides to monitor their biodistribution, physiological processes and biochemical pathways etc [22]. These radionuclides are incorporated into molecules during the radiosynthesis step, generating the radiotracer. PET scans are acquired following the injection of the radiotracer. The radiotracer accumulates in the tissues, and its radionuclide decays by emission of a positron (anti-electron). After travelling at most a few millimeters, a positron will collide with an electron, simultaneously releasing two gamma rays (photons) with energy of $511 \mathrm{keV}$ into opposite directions. These two photons are detected by the PET camera and by collecting a statistically significant number of radioactive events, mathematical algorithms reconstruct a three-dimensional image that shows the distribution of the positron-emitting molecules in the tissues [19]. Single-photon emission computed tomography (SPECT) is also one kind of nuclear medicine tomographic imaging technique using gamma rays which can provide $3 \mathrm{D}$ information as PET as well. Normally, a marker radioisotope could be attached to an nanoparticles or antibody. After being administered in vivo, the gamma-emission of the isotope from the radiolabeled materials allows the drug to be seen by a gamma-camera. SPECT can generate a true 3D image, which permit accurate localization of organs. Therefore, it can be used to provide information about localized function in internal organs, such as functional cardiac or brain imaging [11].

\subsection{PET images guided biodistribution and pharmacokinetic study of theranostics}

Targeted therapeutic and diagnostic nanocarriers functionalized with antibodies, peptides or other targeting ligands that recognize over-expressed receptors or antigens on tumor cells have potential in the diagnosis and therapy of cancer. Biodistribution study of therapeutic antibodies by PET methodology could quantitatively measured by radiotracer in organs/tissues. Biodistribution and pharmacokinetics could also be recorded by blood sampling at predetermined time points, preferably associated with radiometabolite evaluation with respect to time [23]. PET images were used from heart to determine blood concentrations. Briefly, nude mice bearing human colon tumors (LS-174T) were injected intravenously with a chimeric ${ }^{124}$ I anti-CEA mAb (cT84.66) and imaged serially 1 hour to 7 days postinjection. The results showed that PET blood curves agreed well with direct measurements within $12 \%$ at all time points. The 4.2-day half-life of ${ }^{124} \mathrm{I}$ favors its use for PET study of monoclonal antibodies (mAbs). Therefore, quantitative biodistribution measurements could be achieved from tumor-bearing mice by using ${ }^{124} \mathrm{I}$ anti-CEA mAbs with standard microPET acquisition and processing techniques. Although from the ROI of heart on the PET images, the blood concentrations can be calculated, most of the case, the pharmacokinetic study was done by withdrawing blood sample and analyzing them with radioassay or ELISA [24].

The distribution of monoclonal antibodies is determined by the rate of extravasation in tissue, the rate of distribution within tissue, binding affinity in tissue and the rates of elimination from tissue, route of injection, species etc. Although PET imaging has been applied in the study of Integrin aV $\beta 3$ expression or peptide antagonist [25-27], the mAbs are large molecules with MW of about $150 \mathrm{kDa}$, it seems to be very slow for them to diffuse across vascular endothelial cells. The movement of antibody into tissue by convective transport is thought to be the primary mechanism for the distribution in tissue [28]. The transport is mainly determined by the movement rate of fluid from blood to tissue and by the sieving effect of paracellular pores in the vascular endothelium. Normally, the biodistribution of drugs requires sacrificing a number of rodents and quantitating the tissue concentration by HPLC, ELISA or by counting the radioactivity emitting from the tissue. MicroPET imaging provides a non-invasive way of quantitation of the concentration of mAbs in tissues, especially useful for monitoring the kinetics in target organ such as tumor.

For instance, a chimeric anti-CD105 monoclonal antibody, TRC105, was conjugated to 1,4,7,10-tetraazacyclododecane-1,4,7,10-tetraacetic acid (DOTA) and labeled with (64)Cu. Serial PET imaging revealed that the 4T1 tumor uptake of the tracer was $8.0 \pm 0.5,10.4 \pm 2.8$, and $9.7 \pm 1.8 \% \mathrm{ID} / \mathrm{g}$ (percentage of injected dose per gram tissue) at 4, 24, and $48 \mathrm{~h}$ post-injection, respectively, higher than most organs at late time points which provided excellent tumor contrast. Biodistribution data as measured by gamma counting were consistent with the PET findings [11]. Non-invasive PET imaging of vascular en- 
dothelial growth factor (VEGF) expression using radiolabeled bevacizumab has been investigated in ovarian cancer models [29]. Small-animal PET and microCT images were used to calculate tumor uptake and compared with ex vivo biodistribution at $168 \mathrm{~h}$ after injection. The tumor accumulation of ${ }^{89} \mathrm{Zr}$ labeled bevacizumab was calculated to be $7.38 \% \mathrm{ID} / \mathrm{g}$ using small-animal PET imaging, which is equivalent to ex vivo biodistribution studies. Liver and spleen were the other organs that uptake tracer bevacizumab. This project demonstrated the quantization of radio-labeled bevacizumab in tumor using non-invasive imaging is convenient, which was also validated by ex vivo biodistribution studies. Since VEGF is a major angiogenic factor responsible for the development of tumor vasculature, the expression level of VEGF could be regarded as a factor indicating disease progress. PET imaging were performed in mice bearing human colorectal cancer (HT29) xenografts to investigate the in vivo biodistribution of VEGF after injection of ${ }^{64} \mathrm{Cu}$-DOTA-bevacizumab. The accumulation of ${ }^{64} \mathrm{Cu}$-DOTA -bevacizumab in the tumor was 22.7 $\% \mathrm{ID} / \mathrm{g}, 24 \% \mathrm{ID} / \mathrm{g}, 19.0 \% \mathrm{ID} / \mathrm{g}$ at 24,48 and $72 \mathrm{~h}$, respectively, which was significantly correlated with VEGF expression as measured by western blot $(\mathrm{q}=$ 0.81, $\mathrm{P}=0.004)$ [30].

Currently, the PK and biodistribution were also conducted not only for antibodies, but also for antibody or peptide functionalized theranostics. For example, Dr. Cai $\mathrm{W}$ etc. employed PET imaging using (66) $\mathrm{Ga}$ as the radiolabel to quantitatively evaluate the pharmacokinetics and tumor targeting efficacy of nano-graphene. Nano-graphene oxide (GO) sheets with covalently linked, amino group-terminated six-arm branched polyethylene glycol (PEG; $10 \mathrm{kDa}$ ) chains were conjugated to NOTA (1,4,7-triazacyclononane-1,4,7-triacetic acid, for (66)Ga-labeling) and TRC105 (an antibody that binds to CD105). TRC105-conjugated GO was specific for CD105 in cell culture. (66)Ga-NOTA-GO-TRC105 and (66)Ga-NOTA-GO exhibited excellent stability in complete mouse serum. In 4T1 tumor-bearing mice, these GO conjugates were primarily cleared through the hepatobiliary pathway. (66)Ga-NOTA-GOTRC105 accumulated quickly in the 4T1 tumors and tumor uptake remained stable over time $(3.8 \pm 0.4,4.5$ $\pm 0.4,5.8 \pm 0.3$, and $4.5 \pm 0.4 \% \mathrm{ID} / \mathrm{g}$ at $0.5,3,7$, and $24 \mathrm{~h}$ post-injection respectively; $\mathrm{n}=4$ ). PET imaging successful demonstrated the in vivo tumor targeting property with GO, showing them to be suitable nanoplatforms for future biomedical research such as cancer theranostics [31].

Andresen TL et al covalently attached octreotate (TATE) to the distal end of DSPE-PEG(2000) on
PEGylated liposomes with an encapsulated positron emitter (64)Cu that can be utilized for PET imaging. TATE are somatostatin peptide analogs, can target Somatostatin receptors (SSTRs) that are over-expressed in neuroendocrine tumors (NETs). They used PET imaging to investigate the biodistribution and pharmacokinetics of the (64)Cu-loaded PEGylated liposomes with and without TATE. They found that the presence of TATE on the liposomes resulted in a significantly faster initial blood clearance in comparison to control-liposomes without TATE. PEGylated liposomes with or without TATE accumulated at significantly higher quantities in NETs (5.1 \pm 0.3 and $5.8 \pm 0.2 \% \mathrm{ID} / \mathrm{g}$, respectively) than the free peptide (64)Cu-DOTA-TATE $(1.4 \pm 0.3 \% \mathrm{ID} / \mathrm{g}) \quad 24 \mathrm{~h}$ post-injection. Importantly, (64)Cu-loaded PEGylated liposomes with TATE showed significantly higher tumor-to-muscle $(\mathrm{T} / \mathrm{M})$ ratio $(12.7 \pm 1.0)$ than the control-liposomes without TATE $(8.9 \pm 0.9)$ and the (64)Cu-DOTA-TATE free peptide (7.2 \pm 0.3$)$. The higher T/M ratio of the PEGylated liposomes with TATE suggests some advantage of active targeting of NETs, although no absolute benefit in tumor accumulation over the non-targeted liposomes was observed. The results showed that (64)Cu-loaded PEGylated liposomes with TATE conjugated to the surface could be promising new imaging agents for visualizing tumor tissue and especially NETs [32].

\subsection{SPECT images guided biodistribution and pharmacokinetics study of theranostics}

Single-photon emission computed tomography (SPECT) is a nuclear medicine tomographic imaging technique using gamma rays. Normally, a marker radioisotope could be attached to nanoparticles or antibody. After being administered in vivo, the gamma-emission of the isotope from the radiolabeled nanoparticles or antibody allows the drug to be seen by a gamma-camera.

Since the source of SPECT images are gamma ray emissions, the radioisotope decays and emits gamma rays, which can be detected by a gamma camera to obtain 3D images, which permit accurate localization of organs [33]. This information is typically presented as cross-sectional slices through the patient, but can be freely reformatted or manipulated as required. Therefore, it can be used to provide information about localized function in internal organs, such as functional cardiac or brain imaging. For example, 3D acquisition SPECT of the liver was performed to investigate the correlation between tumor accumulation of In-111-bevacizumab and VEGF-A expression in patients with colorectal liver metastases [34].

Although heart has been used as an organ in PET 
imaging to estimate blood concentration, for pharmacokinetic study using SPECT imaging, the commonly used method is to collect blood samples from patients or animals at timed intervals after injection of the radioisotope-labeled $\mathrm{mAb}$ [23]. The major advantages of radionuclide-based molecular imaging techniques (SPECT and PET) over other modalities (e.g. optical and MRI) are that they are very sensitive (down to the picomolar level), quantitative, and there is no tissue penetration limit. However, one disadvantage is that the resolution (typically $>1 \mathrm{~mm}$ ) of either SPECT or PET is not as high as the other imaging modalities such as MRI. In most cases, the purpose of labeling the nanoparticle with a radionuclide was for the non-invasive evaluation of its biodistribution, pharmacokinetic properties, and/or tumor-targeting efficacy with SPECT or PET [35, 36]. Radiolabeling of nanoparticles was also applied for PK and biodistribution studies for the purpose of theranostics. Dr. Helbok et al radiolabeled DTPA-derivatized lipid-based nanoparticles including including (111)In and (99m)Tc for SPECT and PET images with therapy in vitro and in vivo. Their study showed that PEGylated DTPA-nanoparticles were suitable for targeting applications. Biodistribution in Lewis rats revealed no significant differences between nanoparticles in terms of DTPA loading and particle composition; however, different uptake patterns were found between the radionuclides used [37]. Dr. DeNardo et al applied 111In-labeled, chimeric L6 (ChL6) monoclonal antibody linked iron oxide (IO) nanoparticles for studies on pharmacokinetics, tumor uptake, and therapeutic efficacy in athymic mice bearing human breast cancer tumors. The 111In-labeled ChL6 was conjugated with carboxylated polyethylene glycol (PEG) on dextran-coated iron oxide nanoparticles with one to two ChL6 antibodies per nanoparticle (Fig 2). Inductively heating the nanoparticle by externally applied alternating magnetic field caused tumor necrosis after $24 \mathrm{~h}$ of therapy. After nanoparticle injection, SPECT imaging was carried out to quantify the nanoparticle uptake in the tumor, which was about 14 percentage injected dose per gram (\%ID/g) at $48 \mathrm{~h}$ post-injection. A delay in tumor growth occurred after the alternating magnetic field treatment, which was statistically significant when compared with the untreated group [38, 39]. Dr. Misri and colleagues also applied SPECT imaging for mesothelin expressing in tumors by evaluation of 111In labeled antibodies. Their findings indicated that the antimesothelin antibody $\mathrm{mAbMB}$ may be developed into a diagnostic agent for imaging mesothelin-expressing cancers [40]. Dr. Wu et al also successfully applied gamma scintigraphy by pulsincap capsule in the gastrointestinal tract of dogs by to find out the desintegration and transit behavior of Tetramethylpyrazine phosphate. Their SPECT images confirmed the time controlled capsule releasing in the gastrointestinal delivery in dog at the expected time schedule [41].

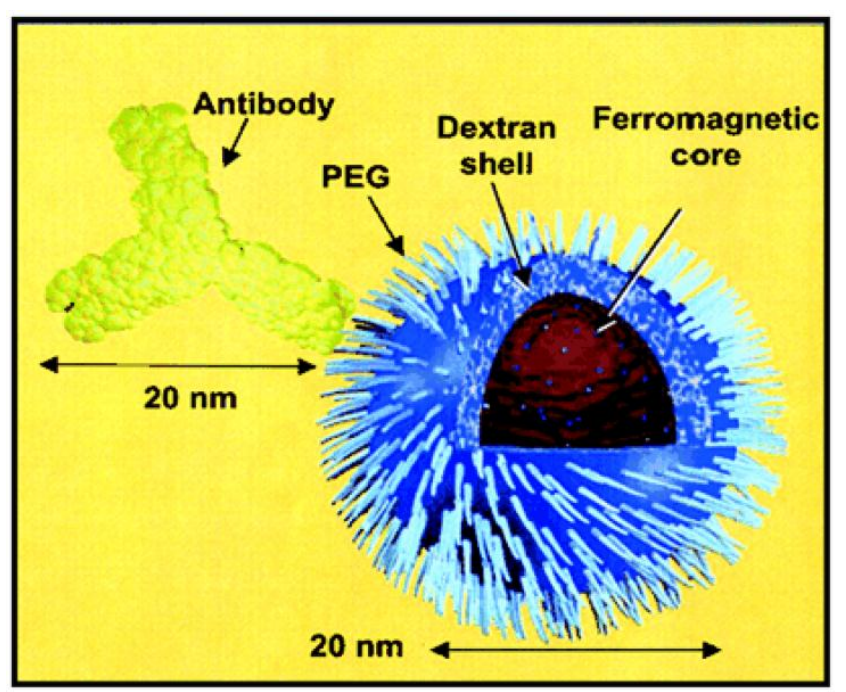

Figure 2. Simple radioimmuno-nanoparticles diagram of a BNF-type particle coated with PEG to link II IIn-ChL6. The particle core has ferromagnetic property. Adapted from [39].

\section{Combination of PET and SPECT with CT, MR or NIRF images guided biodis- tribution and pharmacokinetic studies of theranostics}

Imaging modalities such as CT and PET have been applied sequentially in the diagnosis and staging of disease and in monitoring the effects of therapy for several years. Although PET offers high sensitivity in terms of molecular concentrations and it is possible to measure picomolar amounts of a PET radiopharmaceutical in a tissue. With a three dimentional images obtained by PET, establishing a ROI (region of interest) and set the location of the organs or tissues in the images is of critical importance. Since accurate anatomic localization of functional abnormalities seen on PET scans is well known to be challenging because of the lack of detailed, high-resolution anatomy, the fusion of PET with CT in one camera unit (PET/CT) or fusion of PET image with MR images provides a merged image where not only the physiological status but also the anatomical details may be seen on the same image, which opens new ways of diagnostics and quantitation [42-46]. Indeed, in many cases anatomic imaging is used exclusively, although functional imaging with PET is fulfilling an increasingly 
important role in the staging and therapy monitoring processes, particularly when the CT scan is equivocal. This situation changed dramatically with the recent introduction of the combined PET/CT scanner, an approach that solves the fusion problem through hardware rather than software. This device provides a medical imaging department with the capability to acquire accurately aligned anatomic and functional images for a patient from a single scanning session [47]. In another word, anatomical/functional imaging techniques help to identify the exact location of the signals. Since the patient remains positioned on the same bed for both imaging modalities, temporal and spatial differences between the two sets of images are minimized.

Schneider DW et al used whole-body small-animal PET to compare the bisodistribution and tumor localization of ${ }^{86} \mathrm{Y}$ or ${ }^{111} \mathrm{In}$-labeled antibody formats, derived from a single antimindin/RG-1 mAb in a prostate tumor model. The images were colocalized with simultaneous micro-CT imaging. The antibody accumulation in the tumor correlated with molecular size. The larger antibody formats (IgG and miniantibody) gave higher tumor uptake levels than did the smaller formats (diabody and scFv). These larger formats may be more suitable for radioimmunotherapy applications. The smaller formats were rapidly cleared from circulation, and the diabody, which accumulated in the tumor, may be more suitable for radiodiagnostic applications. The combination of PET with CT provides easier localization of tissues $[34,48]$.

PET is sensitive in the process of detecting and quantifying a low abundance molecular target in cells while MRI is capable of providing exquisite anatomy for organs. Thus, an integrated PET/MRI system will permit the simultaneous acquisition of several parameters in highly accurate spatial and temporal registration. Quantitative values from PET of a large number of biological parameters are complemented by the high-resolution information provided by MRI (in the micromolar range) to yield complementary information. An MRI-based method has the potential to improve PET image quality, increasing its reliability, reproducibility, and quantitative accuracy [49]. PET data and MRI data have been combined for monitoring the drug anticancer effect [50], the response of patients to antibody treatment [51] as well as for detection of lymph node metastases [52], which is very important for the improvement of tumor detection and for the guide of mAb-based therapy.

Overall, accurate quantization of PET tracer uptake levels in tumors is important for staging and monitoring response to treatment. The combination with anatomical information obtained from CT will improve quantization of tumor values in terms of bias and variance [53]. Using appropriate software to fuse PET and CT image together and accurately quantify the tumor uptake level of tracer is of critical value. There is PET/CT scanner available for clinical study for the [54-56]. However, the integrated equipment which combines microPET and contrast-enhanced microCT for small animal study is still at the starting beginning stage $[57,58]$.

PET and NIRF imaging was also combined to study the ex vivo biodistribution 64Cu-DOTA-NuB2-Alexa Fluor 750 in CD20 positive Raji lymphoma-bearing mice. Raji tumor showed significantly higher uptake of DOTA-NuB2-Alexa Fluor 750 than that of DOTA-Alexa Fluor $750(p<0.05)$. Significant correlation was obtained between the organ-to-muscle ratios measured by the radioactivity and fluorescence intensity [59]. In Xie's work, iron oxide nanoparticles with triple functional of PET/NIRF/MRI were applied for the in vivo biodistribution study. To assess the biophysical characteristics of this novel nanosystem, the HSA coated IONPs (HSA-IONPs) were dually labeled with ${ }^{64} \mathrm{Cu}$-DOTA and Cy5.5, and tested in a subcutaneous U87MG xenograft mouse model. In vivo PET/near-infrared fluorescence (NIRF)/MRI tri-modality imaging, and ex vivo analyses and histological examinations were carefully conducted to investigate the in vivo behavior of the nanostructures. This nanosystem is well-suited for dual encapsulation of IONPs and drug molecules, with the compact HSA coating, the HSA-IONPs manifested a prolonged circulation half-life; more impressively, they showed massive accumulation in lesions, high extravasation rate, and low uptake of the particles by macrophages at the tumor area [60].

SPECT has been combined with MRI to investigate the pharmacokinetics and biodistribution. For example, 99mTc-labeled superparamagnetic iron oxide nanoparticles were employed for multimodality SPECT/MRI of sentinel lymph nodes. In Dr. Madru's work, Nanoparticles with a solid iron oxide core and a polyethylene glycol coating were labeled with $(99 \mathrm{~m}) \mathrm{Tc}$. Wistar rats was injected subcutaneously and sacrificed $4 \mathrm{~h}$ after injection. Animals were imaged with SPECT/MRI and the microdistribution within the lymph nodes was studied with digital autoradiography. The result showed that the accumulation of $(99 \mathrm{~m})$ Tc-labeled superparamagnetic iron oxide nanoparticles (as the percentage injected dose $/ \mathrm{g}$ [\%ID/g]) in the lymph nodes was $100 \% \mathrm{ID} / \mathrm{g}$, whereas in the liver and spleen it was less than 2 $\%$ ID/g. Digital autoradiography images revealed a 
nonhomogeneous distribution of $(99 \mathrm{~m}) \mathrm{Tc}$ - labeled superparamagnetic iron oxide nanoparticles within the lymph nodes; nanoparticles were found in the cortical, subcapsular, and medullary sinuses [61].

\section{The optical imaging guided biodistri- bution and pharmacokinetics study of theranostics}

Optical imaging is a photonic technique which applied in the area of imaging, describing the behavior of visible, ultraviolet, and infrared light used in imaging $[62,63]$. These optical techniques, such as bioluminescence and fluorescence, are emerging as powerful new modalities for molecular imaging in disease diagnosis and therapy. Optical imaging offers higher sensitivity and temporal resolution than PET at the same spatial resolution in small animals, but unlike PET is limited to a few centimeters of tissue depth [35]. The bioluminescence and fluorescence can be obtained from dyes, specific metal materials, and biological resources etc and already used in diagnosis and therapy in research and clinic [64-66]. With the help of combing of innovative molecular biology and chemistry, scientists have developed optical methods for imaging a variety of cellular and molecular processes in vivo, including the receptor-ligand interaction, tumor targeting, bio-distribution of proteins such as antibody, etc. Whereas optical imaging has been used primarily for research in small-animal models, in some specific area optical imaging may have the possibility to translate into clinical medicine. The advantages of optical imaging for assessing antibody pharmacokinetics in vivo are the conjugation chemistry, cost, and high-speed throughput. The fluorophore conjugation chemistry is often carried out with a commercially available kit and can be performed in most laboratories without special license, equipment, hazardous precautions, or specific training. The detection systems are generally 30 to $50 \%$ as expensive as those for small-animal SPECT or PET. Planar fluorescence images are generated within seconds or minutes, typically with a photograph overlay. Optical imaging experiments also can accommodate four to five animals per scan, allowing an efficient researcher to perform $100+$ animal images per hour, whereas PET and SPECT imaging is often limited to one to two animals per 10 to $30 \mathrm{~min}$ scan. Such throughput and cost combined with expected improvements in quantification from advances in instrumentation provide an exciting outlook for this preclinical fluorescence imaging approach [67].

A key challenge for pharmacists and clinic doctors is defining the disposition of drugs such as mon- oclonal antibody and pharmacokinetic property in whole animals or patients. Ongoing discoveries of these potential target molecules in disease also drive pharmaceutical and biotechnology companies to find leading agents that selectively regulate these molecular pathways in vitro and in vivo. Among all the existing techniques, optical imaging including bioluminescence and fluorescence, are emerging as key techniques to meet these challenges and advance molecular imaging in preclinical research and patient care. Bioluminescence is the process of light emission in living organisms, it is a chemical process wherein light is emitted during the interaction of a protein produced in engineered cells and an administered substrate, for example firefly luciferase and luciferin [68]. Bioluminescence imaging is a technology developed over the past decade that allows for the noninvasive study of ongo-ing biological processes in small laboratory animals. Bioluminescence imaging utilizes native light emission from one of several organisms which contain bioluminescence. Fluorescence is the emission of light by a substance that has absorbed light or other electromagnetic radiation with a different wavelength. It is a form of luminescence. Fluorescence imaging uses probes that emit light (fluorescence) after the excitation at a fluorophore-specific wavelength. In most cases, emitted light has a longer wavelength, and therefore lower energy, than the exciting source, known as the Stokes shift [69]. Dr. Wu and colleagues applied NIR dye labeled Bevacizumab antibody to find out the pharmacokinetics, lymph Node Uptake and mechanistic PK behavior model after i.v. and s.c. administration in mice [70, 71]; they also found an interesting phenomena that after s.c. administration in mice, the lymph node uptake of proteins in mice had a molecular weight dependence by the way of fluorescence imaging [72]. This finding may provide quite useful aim for the future protein biodistribution and pharmacokinetic studies.

The most important application of optical imaging in the biological field is early cancer detection or diagnosis in preclinic and clinic studies, they provided a very useful alternative modality for tumor imaging [73]. Dr. Hülper P and his colleagues published their finding of tumor localization of nti-TGF- $\beta$ antibody and its effects on gliomas. The orthotopic gliomas beared mice was subcutaneous injected with AF680-labeled 1D11, a pan-neutralizing TGF- $\beta$ antibody, and used for the bio-distribution study via in vivo imaging and fluorescence microscopy. With the combination of results from direct tumor size measurement, H\&E staining and immune-histochemistry, it demonstrate that TGF- $\beta$ plays different roles in 
combating the tumor depending on subcutaneous versus orthotopic implantation site [74]. Besides the monoimaging principle, combining two or more different imaging modalities in the same agent can be of considerable value in molecular imaging. By binding a biotinylated anti-Her2 Herceptin antibody to provide tumor targeting, a biotinylated DOTA chelator labeled with ${ }^{111} \mathrm{In}$ and a biotinylated Cy5.5 fluorophore to a streptavidin nanoparticle, the imaging results show high tumor accumulation and strong tumor-to-normal tissue contrast by both fluorescence and nuclear imaging. The subsequent biodistribution study confirmed the specific tumor accumulation of radioactivity at $40 \mathrm{~h}$ was $21 \mathrm{ID} \% / \mathrm{g}$ and therefore much higher than all other tissues including liver, heart, kidney, spleen, and muscle that accumulated 8.7, 2.5, 6.9, 7.2, and $1.9 \mathrm{ID} \% / \mathrm{g}$, respectively [75]. In Cohen's work, IRDye800CW was coupled with monoclonal antibodies of cetuximab and bevacizumab with ${ }^{89} \mathrm{Zr}$ multi-labeling for optical imaging of tumor targets. The biodistribution studies showed that blood clearance was enhanced and live uptake was increased at 24 to 72 hours post injection when 2 or more eq of dye had been coupled to mAbs. This study realized that after labeling with IRDye800CW, optical imaging can be used for accurate quantification of the biodistribution of mAbs (Figure 2) [76].

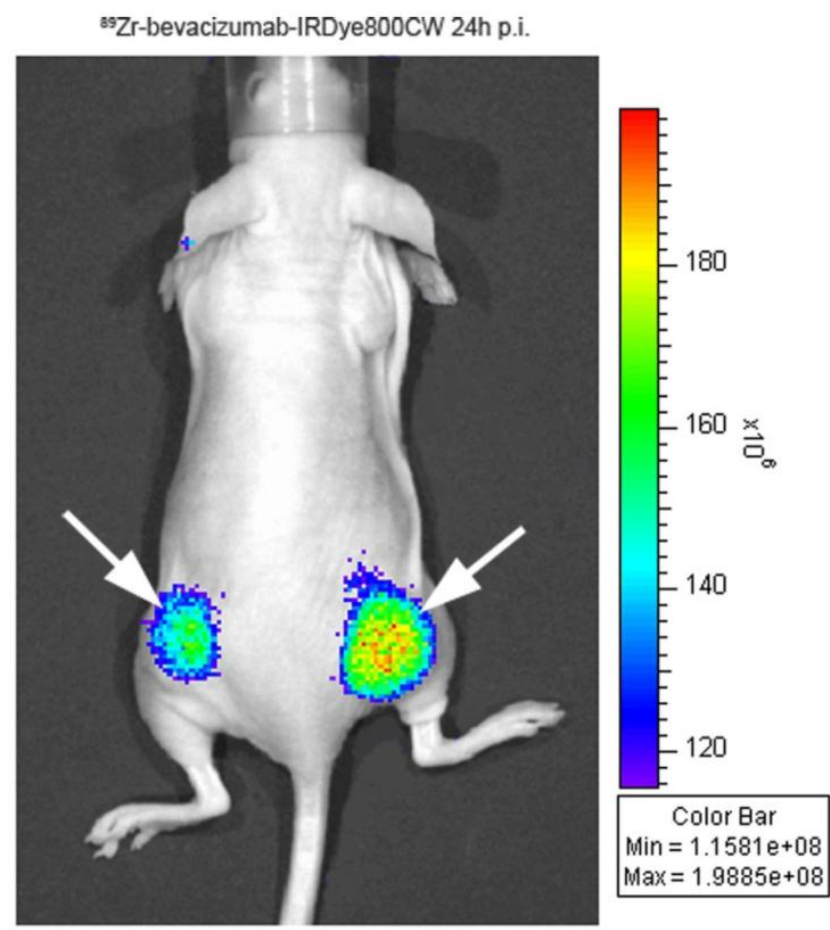

Figure 3. Optical imaging with ${ }^{89} \mathrm{Zr}$-bevacizumab-IRDye800CW in a tumor-bearing mouse. Adapted from [76].
In the area of cancer diagnosis, organic dye and semiconductor nanocrystals (so called quantum dots (QDs) or quantum rods (QRs) attract attentions because of its unique optical properties to be fluorescent probes or luminescent nanoprobes for biological applications, ranging from immunoassays to live cell and tissue imaging, especially in cancer detection [77-82]. Such nanomaterials have their advantages over traditional fluorescent probes such as organic dyes and fluorescent proteins [79, 83]. For example, they have robust photochemical stability, high quantum yield, and excellent resistance to chemical and photochemical degradation, as well as size-tunable photoluminescence that ranges from visible to near-IR with sharp spectral bands [84]. Also, QDs with different emission colors can be simultaneously excited with a single light source, with minimal spectral overlap, providing significant advantages for multiplexed detection of molecular targets $[85,86]$. Thus, with conjugation with antibody and drug on the same QDs, this kind of theranostic reagent can be used as new type of probes for optical bioimaging and treatment on cancer.

Although there are significant advantages of semi-conductor nanocrystal QDs in the application of optical imaging for cancer detection, the potential toxicity cannot be ignored due to the existing of Cadmium element component inside the particles. In order to alleviate possible toxicity and increase the stability of QDs in biological field, Ding et al successfully employed carboxyl functional Pluronics F127 (F127COOH) into the antibody conjugated QDs for pancreatic cancer detection and obtained satisfied results. They successfully applied anti-mesothelin antibody in vivo for pancreatic cancer detection [87]. The in vitro and in vivo toxicity study showed the low damage to cells and tissues in small animals. Another alternative on optical imaging is using biodegradable polymer encapsulated NIR dye for cancer detection [88]. With proper fluorescent dye or drug encapsulation, this kind of biodegradable copolymer can be conjugated with target ligands or specific antibody for all purpose of tumor targeting. Because of the concerning about the possible toxicity, researchers also generated a "pure" bioconjugated luminescent silicon quantum dots for cancer detection $[89,90]$. This kind of luminescent probe can even used for sentinel lymph node mapping since it is one of the most important site that the metastatic happened [91]. Although the above nanomaterials have their advantages over traditional fluorescent dye probes, batch to batch reproducibility and quantization of the labeled drug still need further investigation. Whether the biodistribution of the labeled therapeutic antibodies is sig- 
nificantly affected by the nanomaterials remains a question to be addressed.

Over the past years, different kinds of optical imaging techniques have been developed for biomedical applications, including various microscopy methods for in vitro and ex vivo applications as well as several methods for in vivo applications such as bioluminescence imaging, fluorescence imaging, ultrasound, dark field, diffused optical tomography, and optical coherence tomography, etc. Not only with help from expertise from studies on materials, various techniques can be used in combination, either simultaneously or sequentially, to provide complementary information from the same cells, tissues, organs, or animals [92-96]. Dr. Chen's lab applied fluorescent Maestro imaging system by real-time video imaging of protease expression in vivo, they found out that PEGylation of a molecular beacon with a specific size PEG can significantly alter the activation properties of the probe targeting matrix metalloproteinases (MMPs). And MMPs are a family of zinc-dependent endopeptidases and represent the most well-known proteases associated with tumorigenesis [97].

Among them, both fluorescence and bioluminescence imaging techniques have been found wide applications for in vivo tumor optical imaging in mouse models and afford convenient, frequent visualization and measurement of tumor biomarkers in a real time, sensitive, and noninvasive way. It also can allow for longitudinal detection of disease progression and therapeutic response in the same animals so as to minimize the subject-to-subject variability and reduce the animal number required by a traditional method. Importantly, such in vivo studies are very valuable for bridging the gap between in vitro and in vivo studies and facilitating preclinical and further translational studies [98].

\section{Images guided engineered nanoparti- cles and antibodies cross blood-brain barrier (BBB)}

The blood-brain barrier (BBB) is a special tight capillaries membrane in CNS that formed from a continuous layer of endothelial cells bound together with tight junctions that allow very little transcellular or pericellular transport of molecules in blood fluid [99]. The BBB is an obstacle for clinicians and complicates the treatment of diseases of the central nervous system (CNS). In order to develop BBB associated theranostic techniques, neuroimaging techniques have become increasingly important in assessing the biologic and physiologic properties of brain tumors and neurologic lesions (11). Although there are many successful imaging platforms including X-ray tomography (CT), optical imaging, positron emission tomography (PET), single-photon-emission computed tomography (SPECT), ultrasound (US), and magnetic resonance imaging (MRI), etc. MRI and combining with US seems the most successful applications in the clinic.

Nanoparticles of course can be employed as effective tool for theranostic purpose. One biocompatible stealth nanoparticles were designed by using microemulsion methodology to optimize BBB circumvention and encapsulate drugs for brain delivery. In vitro and in vivo data showed that the formulation has no effect on BBB barrier integrity and membrane permeability, or alteration of expression of the BBB tight-junction proteins, occludin, and claudin-1 while crossing BBB [100]. This kind of nanoparticles can be applied in theranostic through BBB. Nanoparticles encapsulation or conjugation of transporter substrates have already been used for enhance the access of drugs to the brain. In last decade, nano-sized carrier comprised by polymer, emulsion, liposome, nanocrystals, micelles and etc, are already make great progress in pharmaceutical field for drug targeting and imaging. Dr. Ding and his colleagues applied inorganic gold nanords for siRNA delivery into brain has been succeeded in vitro and in vivo based on their contrast property in cancer detection [64, 101-103]. They also applied fluorescent nanocrystal as theranostic carrier for CNS drug delivery system [104]. Dr. Koffie et al prepared biodegradable nanocarrier systems made up of poly(n-butylcyanoacrylate) dextran polymers coated with polysorbate 80 (PBCA nanoparticles) to deliver BBB-impermeable molecular imaging probes into the brain for targeted molecular neuroimaging. They demonstrated that PBCA nanoparticles using as in vivo targeting of BBB-impermeable contrast agents can deliver BBB-impermeable targeted fluorophores of a wide range of sizes: from 0.5k-Da targeted polar molecules to 150k-Da tagged immune- globulins into the brain of living mice model of Alzheimer's disease. PBCA nanoparticles do not induce nonspecific BBB disruption, but collaborate with plasma apolipoprotein E to facilitate BBB crossing [105]. Dr. Qiao et al conjugated lactoferrin (Lf) with poly(ethylene glycol) (PEG)-coated $\mathrm{Fe}_{3} \mathrm{O}_{4}$ nanoparticle to cross $\mathrm{BBB}$ by receptor-mediated transcytosis via the Lf receptor present on cerebral endothelial cells. The MRI images confirmed that in vivo experiments, Fe3O4-Lf probe exhibited an enhanced effective measure for delivering the nanoparticles across the BBB $[106,107]$.

It is well-known that few of those antibodies can penetrate the shield of tightly packed cells known as 
BBB. Normally, antibody concentrations in the brain are typically about a thousand times lower than in the blood. In the recent finding, Dr. Watts and his collaborators report the design of an antibody that surmounts this obstacle. This antibody had the function of targeting two proteins. One is called $\beta$-secretase 1 , which is a popular target for drugs to treat Alzheimer's disease; the second protein targeted by the antibody is the transferrin receptor, which activates a molecular channel that normally imports iron into the brain. By clinging to this receptor, the antibody is transported into the brain, where it can act against $\beta$-secretase 1 . The double-duty antibody performed well in mouse models of Alzheimer's disease: a day after receiving a single injection of the antibody, concentrations of amyloid- $\beta$ in the brain plummeted by $47 \%[108,109]$.

Lee et al applied peptidomimetic monoclonal antibodies that target peptide transcytosis systems for the BBB in vivo. Murine monoclonal antibodies to the rat transferrin receptor, such as the OX26 monoclonal antibody, are targeted through the BBB on the transferrin receptor in the rat. The capillary depletion technique demonstrated transcytosis of the RI7-217 rat monoclonal antibodies antibody can through the mouse BBB in vivo. The studies indicate rat monoclonal antibodies to the mouse transferrin receptor may be used for brain drug-targeting studies [110]. Dr. Boado and his colleagues used engineered murine monoclonal antibody to the human insulin receptor (HIR) as a brain drug delivery system for transport across the human BBB (Figure 4). The HIRMAb was humanized by complementarity determining region (CDR) grafting on the framework regions (FR) of the human B43 IgG heavy chain and the human REI kappa light chain. The humanized HIRMAb avidly bound to the HIR of isolated human brain capillaries, which are used as an in vitro model system of the human BBB. The humanized HIRMAb was radiolabeled with 125-iodine, and injected intravenously into an adult, anesthetized Rhesus monkey. Brain scanning showed the humanized HIRMAb was rapidly transported into all parts of the primate brain after intravenous administration. The humanized HIRMAb may be used as a brain drug and gene delivery system for the targeting of large molecule therapeutics across the BBB in humans [111].

Figure 4. Film autoradiography of Rhesus monkey brain removed $2 \mathrm{~h}$ after an intravenous administration of [125I]-humanized HIRMAb. Coronal sections through the forebrain (top panel), midbrain (middle panel), and hindbrain/cerebellum (bottom panel) are shown. Adapted from [III].

\section{Conclusions and Perspectives}

Molecular imaging takes advantage of the traditional diagnostic imaging techniques and introduces molecular imaging probes to measure the expression of indicative molecular markers at different stages of diseases. It has been widely reported the importance and advantages of various imaging modelities in the drug development. We summarized the distribution and pharmacokinetics studies using MR imaging, CT, PET imaging, SPECT imaging, optical imaging as well as SPECT etc. Among those imaging modalities, PET, SPECT and MRI imaging has been used in clinical study, while optical imaging is still on the way of getting through; almost all of the studies using optical imaging are in the preclinical status. Non-invasive detection of various molecular markers of diseases can allow for much earlier diagnosis, earlier treatment, and better prognosis that will eventually lead to personalized medicine. For using molecular imaging to investigate the biodistribution and PK properties of theranostics, the following aspects still need further investigation:

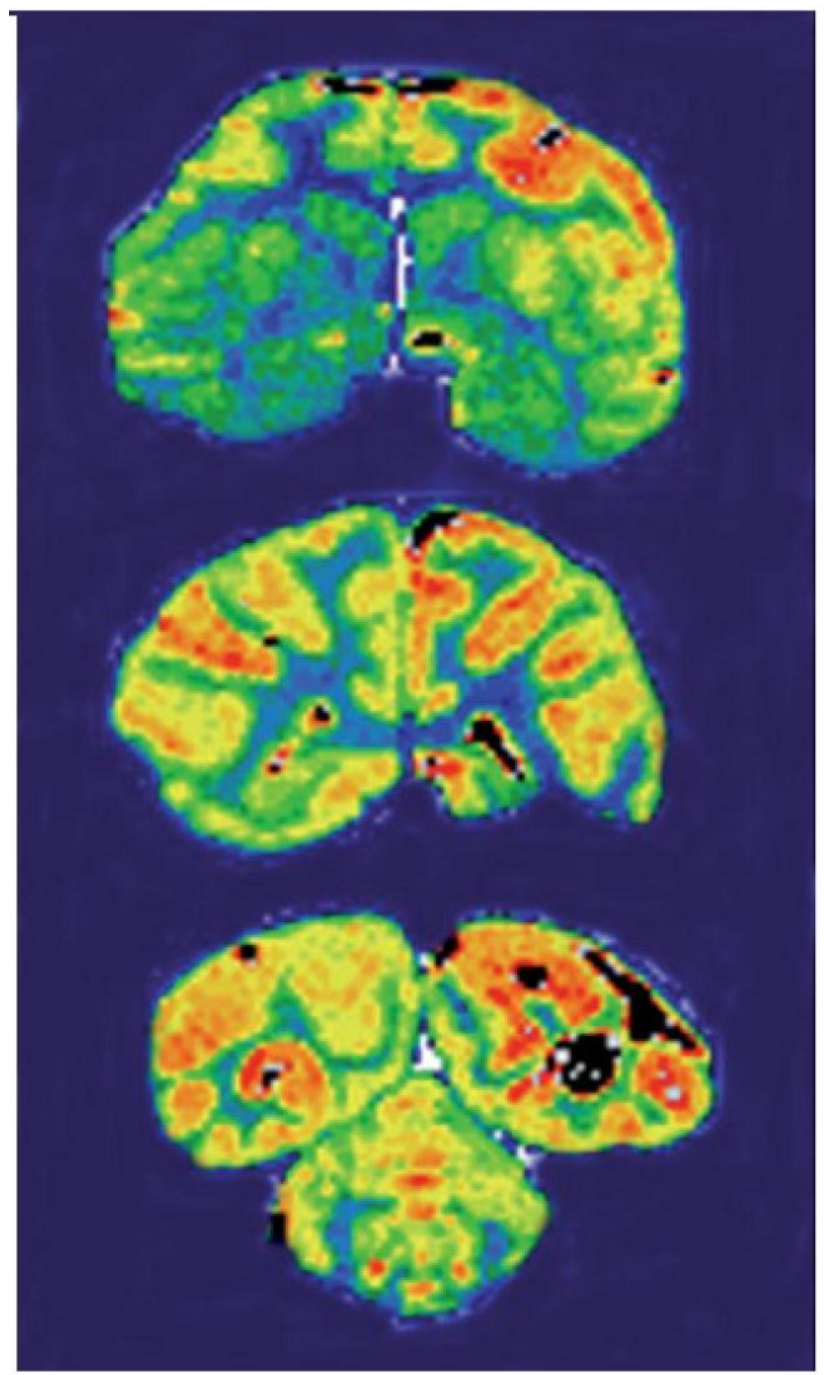


- Major advantages of radionuclide-based molecular imaging techniques (SPECT and PET) over other modalities (optical and MRI) are high sensitivity (picomolar level), quantitation, and non-tissue penetration limitation. However, one disadvantage is that the resolution of either SPECT or PET is not as high as MRI. In most cases, the modalities such as PET or SPECT can be applied for studies of biodistribution, PK study properties, and/or tumor-targeting efficacy in non-invasive image ways.

- Comparing with other medical imaging techniques such as CT, MRI provides good contrast between the different soft tissues of the body, which make it especially useful in imaging brain, muscles, the heart, and cancers. CT is preferable in the application of bones imaging while MRI is good for tissue imaging.

- For both PET imaging and optical imaging, the image only showed the signals from the radioisotope probe or fluorophore probe, while the probe may still attach to the metabolite of drug even after the degradation of the drug. Therefore, the imaging method is not able to distinguish the metabolite or parent drug. Using sodium dodecyl sulfate polyacrylamide gel electrophoresis (SDS-PAGE) or size exclusion HPLC for confirming the stability of the labeled antibody therapeutics may be a good way of providing more information of metabolism. For labeled antibody, due to its therapeutic effect and detective property, the labeled antibody can also be considered as theranostics if it is used in the field of treatment and diagnosis.

- After obtaining biodistribution data using molecular imaging in preclinical study, establishing physiologically based pharmacokinetic model (PBPK) and allometric scaling for translation of animal data to human clinical pharmacology is very important. Further research effort is needed on establishing PBPK model using imaging data.

- For the later clinical application, the absorption, distribution, metabolism/degradation and excretion of theranostics need to be extensively studied. Various imaging modalities mentioned in this review are useful quantitative tools for investigating the above biopharmaceutical properties of theranostis.

\section{Abbreviations}

BBB, blood-brain barrier; CDR, complementarity determining region; $\mathrm{CT}$, computed tomography; DOTA, 1,4,7,10-tetraazacyclododecane-1,4,7,10tetraacetic acid; ELISA, Enzyme-linked immuno- sorbent assay; FR, framework regions; HIR, human insulin receptor; HPLC, high-performance liquid chromatography; Lf, lactoferrin; mAbs, monoclonal antibodies; MRI, magnetic resonance imaging; NETs, neuroendocrine tumors; NIRF, near-infrared fluorescence; PBCA, poly(n-butylcyano- acrylate) dextran polymers coated with polysorbate 80; PBPK, physiologically based pharmacokinetic model; PET, positron emission tomography; ROI, region of interest; SDS-PAGE, sodium dodecyl sulfate polyacrylamide gel electrophoresis; SPECT, single-photon emission computed tomography; TATE, octreotate; US, ultrasound; USPIO, ultrasmall superparamagnetic iron oxide; VEGF, vascular endothelial growth factor.

\section{Competing Interests}

The authors have declared that no competing interest exists.

\section{References}

1. Grosu AL, Weber WA, Franz $M$ et al. Reirradiation of recurrent high-grade gliomas using amino acid PET (SPECT)/CT/MRI image fusion to determine gross tumor volume for stereotactic fractionated radiotherapy. International journal of radiation oncology, biology, physics 2005; 63: 511-519.

2. Hofmann M, Steinke F, Scheel V et al. MRI-Based Attenuation Correction for PET/MRI: A Novel Approach Combining Pattern Recognition and Atlas Registration. Journal of Nuclear Medicine 2008; 49: 1875-1883.

3. Wintermark M, Meuli R, Browaeys P et al. Comparison of CT perfusion and angiography and MRI in selecting stroke patients for acute treatment. Neurology 2007; 68: 694-697.

4. Visser BC, Yeh BM, Qayyum A et al. Characterization of Cystic Pancreatic Masses: Relative Accuracy of CT and MRI. American Journal of Roentgenology 2007; 189: 648-656.

5. Pien $\mathrm{HH}$, Fischman AJ, Thrall JH, Sorensen AG. Using imaging biomarkers to accelerate drug development and clinical trials. Drug Discovery Today 2005; 10: 259-266.

6. Zerizer I, Tan K, Khan S et al. Role of FDG-PET and PET/CT in the diagnosis and management of vasculitis. European Journal of Radiology 2010; 73: 504-509.

7. Rai $\mathrm{P}$, Mallidi S, Zheng $\mathrm{X}$ et al. Development and applications of photo-triggered theranostic agents. Advanced Drug Delivery Reviews 2010; 62: 1094-1124

8. An G, Wu F, Morris ME. 5,7-Dimethoxyflavone and multiple flavonoids in combination alter the ABCG2-mediated tissue distribution of mitoxantrone in mice. Pharm Res 2011; 28: 1090-1099.

9. $\mathrm{Wu} F$, Zhang $Z$, Ding $H$. Simple high-performance liquid chromatographic method for the determination of tetramethylpyrazine phosphate in very small volumes of dog plasma: application to a pharmacokinetic study. J Chromatogr Sci 2006; 44: 13-17.

10. Masutomi T, Onishi H, Takahashi Y, Machida Y. Biodistribution study using Egg Protein ELISA kit after administration of FITC-labeled ovalbumin solution and its double liposomes in the in situ loop method, and its implication in oral immunization. Curr Drug Deliv 2010; 7: 447-452.

11. Hong H, Goel S, Zhang Y, Cai W. Molecular Imaging with Nucleic Acid Aptamers. Current Medicinal Chemistry 2011; 18: 4195-4205.

12. Pickhardt P, Park S, Hahn L et al. Specificity of unenhanced CT for non-invasive diagnosis of hepatic steatosis: implications for the investigation of the natural history of incidental steatosis. European Radiology 2012; 22: 1075-1082.

13. Funkhouser J. Reinventing pharma: The theranostic revolution. Current Drug Discovery 2002; 2: 17-19.

14. Li M KH, et al. Comparison of Two Ultrasmall Superparamagnetic Iron Oxides on Cytotoxicity and MR Imaging of Tumors. Theranostics 2012; 2: 76-85.

15. Ray P DA. Reporter Gene Imaging in Therapy and Diagnosis. Theranostics 2012; 2: 333-334. 
16. Palm S, Enmon RM, Matei $C$ et al. Pharmacokinetics and Biodistribution of 86Y-Trastuzumab for 90Y Dosimetry in an Ovarian Carcinoma Model: Correlative MicroPET and MRI. Journal of Nuclear Medicine 2003; 44: 1148-1155.

17. Wen S, Liu D-F, Liu Z et al. OxLDL-targeted iron oxide nanoparticles for in vivo MRI detection of perivascular carotid collar induced atherosclerotic lesions in ApoE-deficient mice. Journal of Lipid Research 2012; 53: 829-838.

18. Vyjayanti VN, Rao KS. DNA double strand break repair in brain: Reduced NHEJ activity in aging rat neurons. Neuroscience Letters 2006; 393: 18-22.

19. Reddy S, Robinson MK. Immuno-positron emission tomography in cancer models. Semin Nucl Med 2010; 40: 182-189.

20. Voss SD, Smith SV, DiBartolo N et al. Positron emission tomography (PET) imaging of neuroblastoma and melanoma with 64Cu-SarAr immunoconjugates. Proc Natl Acad Sci U S A 2007; 104: 17489-17493.

21. Dearling JL, Voss SD, Dunning $P$ et al. Imaging cancer using PET--the effect of the bifunctional chelator on the biodistribution of a (64)Cu-labeled antibody. Nucl Med Biol 2011; 38: 29-38.

22. Lancelot S, Zimmer L. Small-animal positron emission tomography as a tool for neuropharmacology. Trends Pharmacol Sci 2010; 31: 411-417.

23. Bading JR, Horling M, Williams LE et al. Quantitative serial imaging of an 124I anti-CEA monoclonal antibody in tumor-bearing mice. Cancer Biother Radiopharm 2008; 23: 399-409.

24. Herbertson RA, Tebbutt NC, Lee FT et al. Phase I biodistribution and pharmacokinetic study of Lewis Y-targeting immunoconjugate CMD-193 in patients with advanced epithelial cancers. Clin Cancer Res 2009; 15: 6709-6715.

25. Beer AJ, H K, H-J W, Schwaiger M. PET Imaging of Integrin $\alpha \mathrm{V} \beta 3$ Expression. Theranostics 2011; 1: 48-57.

26. Jacobson OJ, Weiss ID, Szajek LP et al. PET imaging of CXCR4 using copper-64 labeled peptide antagonist. Theranostics 2011; 1: 251-262.

27. Liu S LH, et al. PET Imaging of Integrin Positive Tumors Using $18 \mathrm{~F}$ Labeled Knottin Peptides. Theranostics 2011; 1: 403-412.

28. Wang W, Wang EQ, Balthasar JP. Monoclonal antibody pharmacokinetics and pharmacodynamics. Clin Pharmacol Ther 2008; 84: 548-558

29. Nagengast WB, de Vries EG, Hospers GA et al. In vivo VEGF imaging with radiolabeled bevacizumab in a human ovarian tumor xenograft. J Nucl Med 2007; 48: 1313-1319.

30. Paudyal B, Paudyal P, Oriuchi N et al. Positron emission tomography imaging and biodistribution of vascular endothelial growth factor with 64Cu-labeled bevacizumab in colorectal cancer xenografts. Cancer Sci 2011; 102: 117-121.

31. Hong $\mathrm{H}$, Zhang $\mathrm{Y}$, Engle JW et al. In vivo targeting and positron emission tomography imaging of tumor vasculature with 66Ga-labeled nano-graphene. Biomaterials 2012; 33: 4147-4156.

32. Petersen AL, Binderup T, Jølck RI et al. Positron emission tomography evaluation of somatostatin receptor targeted $64 \mathrm{Cu}$-TATE-liposomes in a human neuroendocrine carcinoma mouse model. Journal of Controlled Release 2012.

33. Pimlott SL, Sutherland A. Molecular tracers for the PET and SPECT imaging of disease. Chemical Society Reviews 2011; 40: 149-162.

34. Schneider DW, Heitner T, Alicke B et al. In vivo biodistribution, PET imaging, and tumor accumulation of 86Y- and 111In-antimindin/RG-1, engineered antibody fragments in $\mathrm{LNCaP}$ tumor-bearing nude mice. J Nucl Med 2009; 50: 435-443.

35. Massoud TF, Gambhir SS. Molecular imaging in living subjects: seeing fundamental biological processes in a new light. Genes \& Development 2003; 17: 545-580.

36. Gambhir SS. Molecular imaging of cancer with positron emission tomography. Nat Rev Cancer 2002; 2: 683-693.

37. Helbok A, Decristoforo C, Dobrozemsky G et al. Radiolabeling of lipid-based nanoparticles for diagnostics and therapeutic applications: a comparison using different radiometals. Journal of Liposome Research 2010; 20: 219-227.

38. DeNardo SJ, DeNardo GL, Miers LA et al. Development of Tumor Targeting Bioprobes (111In-Chimeric L6 Monoclonal Antibody Nanoparticles) for Alternating Magnetic Field Cancer Therapy. Clinical Cancer Research 2005; 11: 7087s-7092s.

39. Natarajan A, Gruettner C, Ivkov R et al. NanoFerrite Particle Based Radioimmunonanoparticles: Binding Affinity and In Vivo Pharmacokinetics. Bioconjugate Chemistry 2008; 19: 1211-1218.

40. Misri R, Saatchi K, Ng SSW et al. Evaluation of 111In labeled antibodies for SPECT imaging of mesothelin expressing tumors. Nuclear Medicine and Biology 2011; 38: 885-896.
41. Fang $\mathrm{Wu}, \mathrm{Hong}$ Ding, Zhang $\mathrm{Z}$. Investigation of the in vivo desintegration and transit behavior of Tetramethylpyrazine phosphate pulsincap capsule in the gastrointestinal tract of dogs by gamma scintigraphy. Journal of Biomedical Engineering 2006; 23: 790-794.

42. Tran L, Huitema AD, van Rijswijk MH et al. CD20 antigen imaging with 124 I-rituximab PET/CT in patients with rheumatoid arthritis. Hum Antibodies 2011; 20: 29-35.

43. Bouchelouche K, Tagawa ST, Goldsmith SJ et al. PET/CT Imaging and Radioimmunotherapy of Prostate Cancer. Semin Nucl Med 2011; 41: $29-44$.

44. Suga K, Yasuhiko K, Hiyama A et al. F-18 FDG PET/CT findings in a patient with bilateral orbital and gastric mucosa-associated lymphoid tissue lymphomas. Clin Nucl Med 2009; 34: 589-593.

45. Bouchelouche K, Capala J, Oehr P. Positron emission tomography/computed tomography and radioimmunotherapy of prostate cancer. Curr Opin Oncol 2009; 21: 469-474.

46. Welch MJ. Potential of labeling monoclonal antibodies for positron emission tomography and magnetic resonance imaging. Acta Radiol Suppl 1990; 374: 129-133.

47. Townsend DW, Carney JPJ, Yap JT, Hall NC. PET/CT Today and Tomorrow. Journal of Nuclear Medicine 2004; 45: 4S-14S.

48. Gonzalez Trotter DE, Manjeshwar RM, Doss M et al. Quantitation of small-animal (124)I activity distributions using a clinical PET/CT scanner. J Nucl Med 2004; 45: 1237-1244.

49. Heiss WD. The potential of PET/MR for brain imaging. Eur J Nucl Med Mol Imaging 2009; 36 Suppl 1: S105-112.

50. Sharma R, Katz JK. Taxotere chemosensitivity evaluation in mice prostate tumor: validation and diagnostic accuracy of quantitative measurement of tumor characteristics by MRI, PET, and histology of mice tumor. Technol Cancer Res Treat 2008; 7: 175-185.

51. Smith TA. Towards detecting the HER-2 receptor and metabolic changes induced by HER-2-targeted therapies using medical imaging. Br J Radiol 2010; 83: 638-644.

52. Borjesson PK, Jauw YW, Boellaard R et al. Performance of immuno-positron emission tomography with zirconium-89-labeled chimeric monoclonal antibody U36 in the detection of lymph node metastases in head and neck cancer patients. Clin Cancer Res 2006; 12: 2133-2140.

53. Alessio AM, Kinahan PE. Improved quantitation for PET/CT image reconstruction with system modeling and anatomical priors. Med Phys 2006; 33: 4095-4103.

54. Minamimoto R, Ito K, Kubota K et al. Clinical Role of FDG PET/CT for Methotrexate-Related Malignant Lymphoma. Clin Nucl Med 2011; 36: 533-537.

55. Freudenberg LS, Jentzen W, Stahl A et al. Clinical applications of 124I-PET/CT in patients with differentiated thyroid cancer. Eur J Nucl Med Mol Imaging 2011; 38 Suppl 1: S48-56.

56. Harms HJ, Knaapen $\mathrm{P}$, de Haan $\mathrm{S}$ et al. Automatic generation of absolute myocardial blood flow images using [15O]H2O and a clinical PET/CT scanner. Eur J Nucl Med Mol Imaging 2011; 38: 930-939.

57. Liang $\mathrm{H}$, Yang $\mathrm{Y}$, Yang $\mathrm{K}$ et al. A microPET/CT system for in vivo small animal imaging. Phys Med Biol 2007; 52: 3881-3894.

58. von Falck $\mathrm{C}$, Rodt $\mathrm{T}$, Halter $\mathrm{R}$ et al. Combined microPET/CT for imaging of hepatocellular carcinoma in mice. Front Biosci 2009; 14: 2193-2202.

59. Paudyal $P$, Paudyal B, Iida $Y$ et al. Dual functional molecular imaging probe targeting CD20 with PET and optical imaging. Oncol Rep 2009; 22: 115-119.

60. Xie J, Chen K, Huang J et al. PET/NIRF/MRI triple functional iron oxide nanoparticles. Biomaterials 2010; 31: 3016-3022.

61. Madru R, Kjellman P, Olsson F et al. 99mTc-Labeled Superparamagnetic Iron Oxide Nanoparticles for Multimodality SPECT/MRI of Sentinel Lymph Nodes. Journal of Nuclear Medicine 2012; 53: 459-463.

62. Weissleder R. Scaling down imaging: molecular mapping of cancer in mice. Nat Rev Cancer 2002; 2: 11-18.

63. Hoppin J, Orcutt KD, Hesterman JY et al. Assessing antibody pharmacokinetics in mice with in vivo imaging. J Pharmacol Exp Ther 2011; 337: 350-358.

64. Ding H, Yong K-T, Roy I et al. Gold Nanorods Coated with Multilayer Polyelectrolyte as Contrast Agents for Multimodal Imaging. The Journal of Physical Chemistry C 2007; 111: 12552-12557.

65. $\mathrm{Xu} \mathrm{Y,} \mathrm{Chang} \mathrm{E,} \mathrm{Liu} \mathrm{H}$ et al. Proof-of-Concept Study of Monitoring Cancer Drug Therapy with Cerenkov Luminescence Imaging. Journal of Nuclear Medicine 2012; 53: 312-317.

66. Wu C, Mino $\mathrm{K}$, Akimoto $\mathrm{H}$ et al. In vivo far-red luminescence imaging of a biomarker based on BRET from Cypridina bioluminescence to an organic dye. Proceedings of the National Academy of Sciences 2009; 106: 15599-15603. 
67. Leblond F, Davis SC, Valdes PA, Pogue BW. Pre-clinical whole-body fluorescence imaging: Review of instruments, methods and applications. J Photochem Photobiol B 2010; 98: 77-94.

68. de Wet JR, Wood KV, DeLuca M et al. Firefly luciferase gene: structure and expression in mammalian cells. Mol Cell Biol 1987; 7: 725-737.

69. Heim R, Cubitt AB, Tsien RY. Improved green fluorescence. Nature 1995; 373: 663-664

70. Wu F, Tamhane M, Morris M. Pharmacokinetics, Lymph Node Uptake, and Mechanistic PK Model of Near-Infrared Dye-Labeled Bevacizumab After IV and SC Administration in Mice. The AAPS Journal 2012; 14: 252-261.

71. Wu F, Bhansali SG, Tamhane M et al. Noninvasive real-time fluorescence imaging of the lymphatic uptake of BSA-IRDye 680 conjugate administered subcutaneously in mice. Journal of Pharmaceutical Sciences 2012; 101: 1744-1754

72. Wu F, Bhansali S, Law W et al. Fluorescence Imaging of the Lymph Node Uptake of Proteins in Mice after Subcutaneous Injection: Molecular Weight Dependence. Pharmaceutical Research 2012; 29: 1843-1853.

73. Zou P, Xu S, Povoski SP et al. Near-infrared fluorescence labeled anti-TAG-72 monoclonal antibodies for tumor imaging in colorectal cancer xenograft mice. Mol Pharm 2009; 6: 428-440.

74. Hulper P, Schulz-Schaeffer W, Dullin C et al. Tumor localization of an anti-TGF-beta antibody and its effects on gliomas. Int J Oncol 2011; 38: 51-59.

75. Liang M, Liu X, Cheng D et al. Multimodality nuclear and fluorescence tumor imaging in mice using a streptavidin nanoparticle. Bioconjug Chem 2010; 21: 1385-1388.

76. Cohen R, Stammes M, de Roos I et al. Inert coupling of IRDye800CW to monoclonal antibodies for clinical optical imaging of tumor targets. EJNMMI Research 2011; 1: 31.

77. Zhang H, Yee D, Wang C. Quantum dots for cancer diagnosis and therapy: biological and clinical perspectives. Nanomedicine (Lond) 2008; 3: 83-91.

78. Hu R, Yong KT, Roy I et al. Functionalized near-infrared quantum dots for in vivo tumor vasculature imaging. Nanotechnology 2010; 21: 145105.

79. Chan WC, Nie S. Quantum dot bioconjugates for ultrasensitive nonisotopic detection. Science 1998; 281: 2016-2018.

80. Yong $\mathrm{KT}$, Ding $\mathrm{H}$, Roy $\mathrm{I}$ et al. Imaging pancreatic cancer using bioconjugated InP quantum dots. ACS Nano 2009; 3: 502-510.

81. Yong KT, Hu R, Roy I et al. Tumor targeting and imaging in live animals with functionalized semiconductor quantum rods. ACS Appl Mater Interfaces 2009; 1: 710-719.

82. Law WC, Yong KT, Roy I et al. Aqueous-phase synthesis of highly luminescent CdTe/ZnTe core/shell quantum dots optimized for targeted bioimaging. Small 2009; 5: 1302-1310.

83. Dubertret B, Skourides P, Norris DJ et al. In vivo imaging of quantum dots encapsulated in phospholipid micelles. Science 2002; 298: 1759-1762.

84. Romero MJ, van de Lagemaat J, Mora-Sero I et al. Imaging of resonant quenching of surface plasmons by quantum dots. Nano Lett 2006; 6: 2833-2837.

85. Michalet X, Pinaud FF, Bentolila LA et al. Quantum dots for live cells, in vivo imaging, and diagnostics. Science 2005; 307: 538-544.

86. Hild WA, Breunig M, Goepferich A. Quantum dots - nano-sized probes for the exploration of cellular and intracellular targeting. Eur J Pharm Biopharm 2008; 68: 153-168.

87. Ding H, Yong KT, Law WC et al. Non-invasive tumor detection in small animals using novel functional Pluronic nanomicelles conjugated with anti-mesothelin antibody. Nanoscale 2011; 3: 1813-1822.

88. Ding H, Yong KT, Roy I et al. Bioconjugated PLGA-4-arm-PEG branched polymeric nanoparticles as novel tumor targeting carriers. Nanotechnology 2011; 22: 165101.

89. Erogbogbo F, Tien CA, Chang CW et al. Bioconjugation of luminescent silicon quantum dots for selective uptake by cancer cells. Bioconjug Chem 2011; 22: 1081-1088.

90. Erogbogbo F, Yong KT, Hu R et al. Biocompatible magnetofluorescent probes: luminescent silicon quantum dots coupled with superparamagnetic iron(III) oxide. ACS Nano 2010; 4: 5131-5138.

91. Erogbogbo F, Yong KT, Roy I et al. In vivo targeted cancer imaging, sentinel lymph node mapping and multi-channel imaging with biocompatible silicon nanocrystals. ACS Nano 2011; 5: 413-423.

92. Moriyama EH, Zheng G, Wilson BC. Optical molecular imaging: from single cell to patient. Clin Pharmacol Ther 2008; 84: 267-271.

93. Wu F, Wuensch SA, Azadniv M et al. Galactosylated LDL nanoparticles: a novel targeting delivery system to deliver antigen to macrophages and enhance antigen specific T cell responses. Mol Pharm 2009; 6: 1506-1517.
94. Liu L, Law WC, Yong KT et al. Multimodal imaging probes based on Gd-DOTA conjugated quantum dot nanomicelles. Analyst 2011; 136: 1881-1886.

95. Law W, Yong K, Roy I et al. Optically and Magnetically Doped Organically Modified Silica Nanoparticles as Efficient Magnetically Guided Biomarkers for Two-Photon Imaging of Live Cancer Cells. J Phys Chem C 2008; 112: 7972-7977.

96. Ding $\mathrm{H}, \mathrm{Wu} F$, Huang $\mathrm{Y}$ et al. Synthesis and characterization of temperature-responsive copolymer of PELGA modified poly(N-isopropylacrylamide). Polymer 2006; 47: 1575-1583.

97. Zhu L XJ, et al. Real-Time Video Imaging of Protease Expression In Vivo. Theranostics 2011; 1: 18-27.

98. Ye $Y$, Chen $X$. Integrin targeting for tumor optical imaging. Theranostics 2011; 1: 102-126.

99. Qiao R, Jia Q, Hüwel S et al. Receptor-Mediated Delivery of Magnetic Nanoparticles across the Blood-Brain Barrier. ACS Nano 2012; 6: 3304-3310.

100. Koziara JM, Lockman PR, Allen DD, Mumper RJ. \&lt;i\&gt;In Situ Blood-Brain Barrier Transport of Nanoparticles. Pharmaceutical Research 2003; 20: 1772-1778.

101. Bonoiu AC, Bergey EJ, Ding $\mathrm{H}$ et al. Gold nanorod-siRNA induces efficient in vivo gene silencing in the rat hippocampus. Nanomedicine 2011; 6: 617-630.

102. Chakravarthy KV, Bonoiu AC, Davis WG et al. Gold nanorod delivery of an ssRNA immune activator inhibits pandemic H1N1 influenza viral replication. Proceedings of the National Academy of Sciences 2010; 107: 10172-10177.

103. Bonoiu AC, Mahajan SD, Ding H et al. Nanotechnology approach for drug addiction therapy: Gene silencing using delivery of gold nanorod-siRNA nanoplex in dopaminergic neurons. Proceedings of the National Academy of Sciences 2009; 106: 5546-5550.

104. Mahajan S. D. RI, Xu GX, Yong K, Ding H., Aalinkeel R., Reynolds J. L., Sykes D. E., Nair B. B., Lin E. Y., Prasad P. N., Schwartz S. A.. Enhancing the delivery of anti retroviral drug "Saquinavir" across the blood brain barrier using nanoparticles. Current HIV Research 2010; 8: 396-404.

105. Koffie RM, Farrar CT, Saidi L-J et al. Nanoparticles enhance brain delivery of blood-brain barrier-impermeable probes for in vivo optical and magnetic resonance imaging. Proceedings of the National Academy of Sciences 2011; 108: 18837-18842.

106. Banks WA. Physiology and pathology of the blood-brain barrier: implications for microbial pathogenesis, drug delivery and neurodegenerative disorders. Journal of Neurovirology 1999; 5: 538-555.

107. Weinstein JS, Varallyay CG, Dosa E et al. Superparamagnetic iron oxide nanoparticles: diagnostic magnetic resonance imaging and potential therapeutic applications in neurooncology and central nervous system inflammatory pathologies, a review. J Cereb Blood Flow Metab 2009; 30: 15-35.

108. Atwal JK, Chen Y, Chiu C et al. A Therapeutic Antibody Targeting BACE1 Inhibits Amyloid- $\beta$ Production in Vivo. Science Translational Medicine 2011; 3: 84ra43

109. Yu YJ, Zhang Y, Kenrick $M$ et al. Boosting Brain Uptake of a Therapeutic Antibody by Reducing Its Affinity for a Transcytosis Target. Science Translational Medicine 2011; 3: 84ra44.

110. Lee HJ, Engelhardt B, Lesley J et al. Targeting Rat Anti-Mouse Transferrin Receptor Monoclonal Antibodies through Blood-Brain Barrier in Mouse. Journal of Pharmacology and Experimental Therapeutics 2000; 292: 1048-1052.

111. Boado RJ, Zhang Y, Zhang Y, Pardridge WM. Humanization of anti-human insulin receptor antibody for drug targeting across the human blood-brain barrier. Biotechnology and Bioengineering 2007; 96: 381-391. 